\title{
Monotherapy for Alopecia Areata: A Systematic Review and Network Meta-Analysis
}

\author{
Aditya K. Gupta ${ }^{a}$ b Jessie L. Carviel ${ }^{a}$ Kelly A. Foley ${ }^{a}$ Neil H. Shear ${ }^{b, c}$ \\ Bianca Maria Piraccini $^{d}$ Vincent Piguet $^{\text {b, e, f }}$ Antonella Tostig \\ ${ }^{a}$ Mediprobe Research Inc., London, ON, Canada; ${ }^{b}$ Division of Dermatology, Department of Medicine, University \\ of Toronto School of Medicine, Toronto, ON, Canada; 'Division of Dermatology, Sunnybrook Health Sciences \\ Centre, Toronto, ON, Canada; ${ }^{d}$ Division of Dermatology, Department of Experimental, Diagnostic and Specialty \\ Medicine, University of Bologna, Bologna, Italy; 'Division of Dermatology, Women's College Hospital, Toronto, ON, \\ Canada; ${ }^{f}$ Division of Infection and Immunity, Cardiff University School of Medicine, Cardiff, UK; ${ }^{9}$ Dermatology and \\ Cutaneous Surgery, University of Miami, Miami, FL, USA
}

\section{Keywords}

Calcineurin inhibitors · Corticosteroids · Minoxidil ·

Prostaglandins - Tofacitinib - Topical immunotherapy

\begin{abstract}
Background: There are many treatments available for alopecia areata; however, none are approved by the US Food and Drug Administration. Thus, there is clinician benefit in efficacy comparison. Methods: A network meta-analysis was used to create direct and indirect comparisons of alopecia areata studies in addition to an inconsistency analysis, risk of bias, and quality of evidence assessment. Results: For mild disease, intralesional corticosteroids were ranked the most likely to produce a response at $78.9 \%$ according to SUCRA (surface under the cumulative ranking curve) followed by topical corticosteroids (67.9\%), prostaglandin analogs (67.1\%), diphenylcyclopropenone (DPCP, 63.4\%), topical minoxidil (61.2\%), and squaric acid dibutylester (SADBE, 35.0\%). In contrast, for moderate to severe disease ( $>50 \%$ scalp hair loss), DPCP was the top-ranked treatment (87.9\%), followed by laser $(77.9 \%)$, topical minoxidil (55.5\%), topical corticoste-
\end{abstract}

\section{KARGER}

(c) 2019 S. Karger AG, Basel

E-Mail karger@karger.com

www.karger.com/sad roids (50.1\%), SADBE (49.7\%), and topical tofacitinib (47.6\%). There were insufficient eligible trials to include oral tofacitinib in the network. Conclusion: Statistically significant evidence is presented for the use of intralesional and topical corticosteroids for treatment of mild disease and DPCP, laser, SADBE, topical minoxidil and topical corticosteroids for moderate to severe disease. Further controlled trials are required to analyze the relative efficacy of oral tofacitinib.

(c) 2019 S. Karger AG, Basel

\section{Introduction}

Alopecia areata (AA) is an autoimmune-mediated nonscarring hair loss disorder that affects approximately $2 \%$ of the population $[1,2]$. Hair loss is the result of an autoimmune process that targets the hair follicle after loss of its immune privilege [3] triggered by viral infections, trauma, hormones, and stress $[4,5]$ in combination with individual genetic predispositions [5-8]. Symptoms range from mild patchy hair loss to severe ( $>50 \%$ scalp involvement) or total hair loss with possible associated 
nail abnormalities. Spontaneous remissions are common, especially in patients with milder symptoms; however, severe symptoms can progress to total scalp hair loss (alopecia totalis, AT) or total hair loss (alopecia universalis, AU) that tend to have a chronic course.

With no clear consensus on optimum therapies and a corresponding lack of approved medications from the US Food and Drug Administration, treatment is not straightforward. Current treatments include steroids (intralesional, topical, and systemic), topical immunotherapy (diphenylcyclopropenone [DPCP], squaric acid dibutylester [SADBE]), topical immunosuppressants (tacrolimus, pimecrolimus), topical minoxidil, and topical prostaglandin analogs (bimatoprost, latanoprost). In addition, new research is ongoing and includes Janus kinase (JAK) inhibitors (tofacitinib, ruxolitinib), laser therapy, and platelet-rich plasma therapy. Therefore, treatment data was analyzed directly and indirectly through use of network meta-analysis to compare efficacies of the various available treatment options for both mild and moderate to severe AA of the scalp.

\section{Materials and Methods}

\section{Literature Search}

PubMed was used with the search term "alopecia areata" and completed on January 16, 2019. Studies were eligible for inclusion if the efficacy of an AA treatment was compared with either a control/placebo treatment or a comparator as well as the number of treatment responders being explicitly stated and defined. A minimum of two such studies was required to include a particular treatment in the analysis. Case studies, studies with only one arm, studies which investigated combination treatments, articles where full text was not available, articles which were not written in English, and articles which did not include detailed methods or results were excluded. Combination treatments were defined as more than one experimental treatment in the same patch. Screening of articles was performed by two authors, J.L.C. and K.A.F.

\section{Data Extraction}

As many studies reported results by number of patches, treatment effects were evaluated based on the number of patches that responded to treatment (response defined as $>75 \%$ regrowth and cosmetic acceptability) at study-defined measurement points. Patches including AT and AU patients were defined by study investigators. Some studies included multiple treatments per patient where different patches of AA received different treatments or were half-head studies where patients served as their own controls. Relapse rates were not included in this analysis as this data was not always available and in some cases patients remained on treatment after study completion. Treatment effects were based on response rates of patients who completed the studies.
Quality of Evidence and Risk-of-Bias Assessment

Two authors (J.L.C. and K.A.F.) assessed evidence quality and risk of bias. Details regarding the quality of evidence and risk-ofbias assessment for this review are provided in online supplementary Appendix S1 (online suppl. materials and methods; for all online suppl. material, see www.karger.com/doi/10.1159/000501940) [9].

\section{Meta-Analysis of Direct Treatment Effects}

For supporting information, see online supplementary material.

\section{Network Meta-Analysis}

Network meta-analysis was used to make mixed comparisons among the therapeutic options and to rank treatments, using the program Aggregate Data Drug Information Software (ADDIS) version 2 [10] (online suppl. Appendix S1; for supporting information, see online suppl. material). Convergence diagnostics and model fit (including heterogeneity) were used to determine that data retrieved from studies of mild disease versus severe disease required separate analysis. Thus, studies were sorted into two groups according to how investigators defined disease severity as best as possible and analyzed: (1) those which described the disease as mild or patchy ( $<3$ patches) and (2) studies which described the disease as severe, affecting $>50 \%$ of the scalp, AT, or AU. Studies containing a mixture of patients (both mild and severe disease) were included in the second analysis of moderate to severe disease as heterogeneity was used to determine the better fit. Nonetheless, this remains a limitation of the analysis. For supporting information on inconsistency analysis, see online supplementary material.

\section{Results}

\section{Clinical Trial Analysis}

A total of 38 trials were included in the analysis, with 11 labeled mild including 968 patches, and 27 labeled moderate to severe including 1,976 patches. Treatment options with sufficient evidence (a minimum of two clinical trials matching the treatment to a control/placebo or comparator) are listed in Table 1 . There were too few eligible studies to include oral tofacitinib in the network. Treatment success was evaluated over a period of 3 months to 1 year for most studies. A network graph summarizes the comparisons (Fig. 1).

\section{Risk of Bias}

Foe supporting information, see online supplementary material.

\section{Quality of Evidence}

Evidence was graded from moderate to very low (online suppl. Table S4; for supporting information, see online suppl. material). Evidence was downgraded due to the prevalence of trials which were not described as randomized. For each comparison, the inclusion of even one nonrandomized trial resulted in a grade of very low-quality evidence. 
Table 1. AA treatments with sufficent evidence for analysis

Treatments analyzed for mild disease

DPCP

Calcineurin inhibitors (pimecrolimus, tacrolimus)

Topical minoxidil (1 and 5\%)

SADBE

Prostaglandin analogs (bimatoprost, latanoprost)

Intralesional corticosteroids (triamcinolone acetonide, betamethasone dipropionate)

Topical corticosteroids (desoximetasone, betamethasone dipropionate, betamethasone valerate, clobetasol propionate, mometasone furoate)
Treatments analyzed for moderate to severe disease

DPCP

Laser

Topical minoxidil $(1,3$, and 5\%)

SADBE

Topical tofacitinib

Topical corticosteroids (clobetasol (di)propionate,

desoximetasone, fluocinolone acetonide, mometasone furoate)

\section{Efficacy of Direct Comparisons of Monotherapy versus} Control

Direct comparisons (meta-analysis and network metaanalysis) of moderate to severe disease with untreated or placebo controls yielded significantly greater odds ratios (OR) of obtaining responders with all treatments (DPCP, topical minoxidil, SADBE, laser, and topical corticosteroids) with the exception of topical tofacitinib (two studies) (Table 2; online suppl. Table S3). Fewer studies per comparison were available in the case of mild disease. Meta-analysis resulted in significantly greater OR in the case of topical corticosteroids (two studies) versus untreated or placebo controls (online suppl. Table S3) while network meta-analysis revealed a significantly greater $\mathrm{OR}$ with the use of intralesional corticosteroids (two studies) (Table 2).

\section{Efficacy of Direct Comparisons of Monotherapy versus}

\section{Comparator}

Direct comparisons (meta-analysis) of mild disease revealed a significantly greater OR of achieving treatment response with intralesional corticosteroids compared with topical corticosteroids (Table 2). In one study, bimatoprost was observed to produce significantly greater OR when compared with topical corticosteroids (Table 2). Differences between all additional comparators (including moderate to severe disease) were found to be not significant.

\section{Mixed Treatment Comparisons}

Mixed treatment comparisons are the network analyses that compare more than two interventions indirectly with at least one pair compared both directly and indirectly [11]. Network results showed no significant differences between comparators for mild disease (all treatments) as well as moderate to severe (topical corticosteroids, topical tofacitinib) disease.

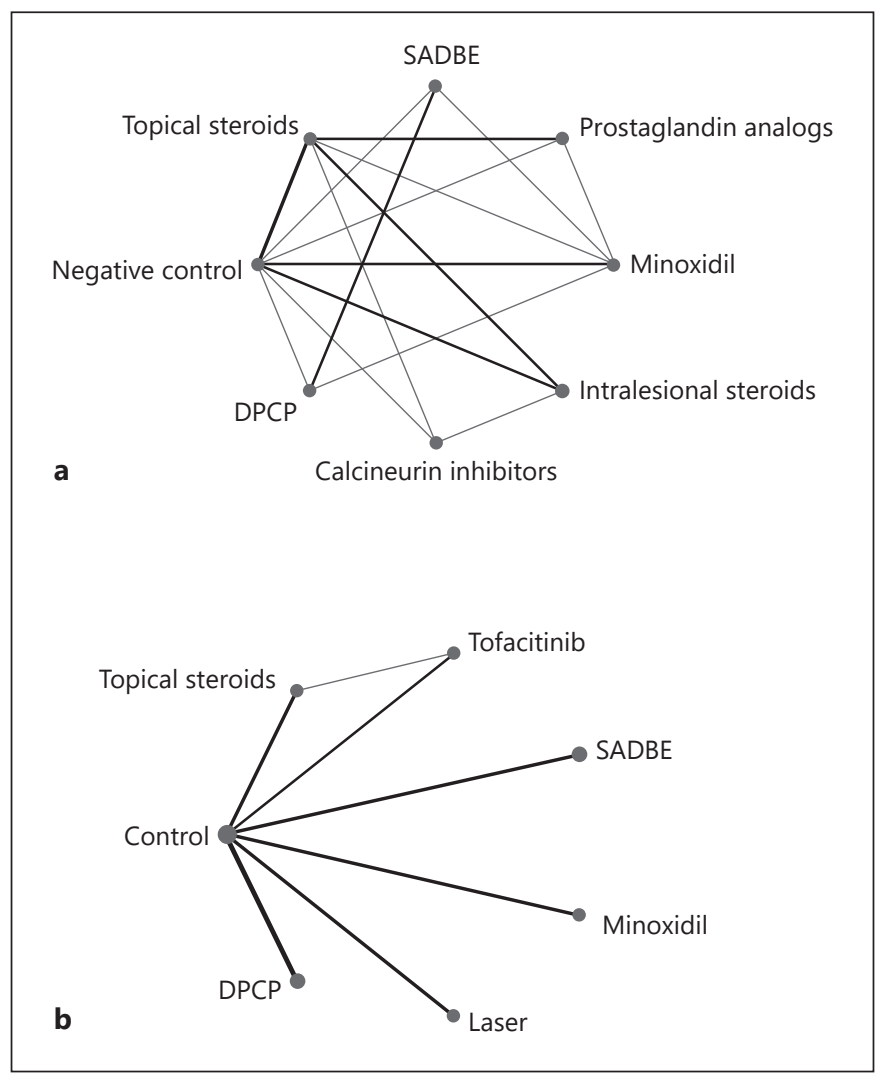

Fig. 1. The network graph shows the evidence network for all selected interventions. The size of an intervention's circle reflects the total number of patches for that intervention. Lines signify that interventions are connected through at least one study, with thicker lines indicating more connecting studies. a Network graph of moderate to severe disease. $\mathbf{b}$ Network graph of mild disease. 
Table 2. Comparison of the included interventions

\begin{tabular}{|c|c|c|c|c|c|c|c|}
\hline \multicolumn{8}{|c|}{ Moderate to severe disease } \\
\hline Control & $\begin{array}{l}249.211 \\
(5.357,295,374.700) \\
\text { Topical corticosteroids }\end{array}$ & $\begin{array}{l}59.734 \\
(1.560,4,295.830) \\
0.241 \\
(0.000,56.741) \\
\text { SADBE }\end{array}$ & $\begin{array}{l}40.654 \\
(0.157,77,419.967) \\
0.152 \\
(0.000,111.286) \\
0.681 \\
(0.001,3,136.930) \\
\text { Tofacitinib }\end{array}$ & $\begin{array}{l}952.795 \\
(20.342,673,336.174) \\
3.996 \\
(0.002,5,142.528) \\
16.192 \\
(0.071,33,057.376) \\
23.924 \\
(0.009,109,316.213) \\
\text { Laser }\end{array}$ & $\begin{array}{l}3,324.585 \\
(81.990,1,079,490.793) \\
12.271 \\
(0.010,7,003.043) \\
54.554 \\
(0.260,54,339.137) \\
75.001 \\
(0.040,219,476.402) \\
3.037 \\
(0.005,2,193.821) \\
\text { DPCP }\end{array}$ & $\begin{array}{l}105.541 \\
(1.659,14,404.559) \\
0.411 \\
(0.000,134.788) \\
1.673 \\
(0.006,841.512) \\
2.631 \\
(0.001,3,698.194) \\
0.107 \\
(0.000,40.189) \\
0.032 \\
(0.000,8.866) \\
\text { Minoxidil }\end{array}$ & \\
\hline \multicolumn{8}{|c|}{ Mild disease } \\
\hline Control & $\begin{array}{l}12.102 \\
(0.646,639.445) \\
\text { Topical corticosteroids }\end{array}$ & $\begin{array}{l}18.791 \\
(0.186,7,360.624) \\
1.551 \\
(0.019,176.938) \\
\text { Prostaglandin analog }\end{array}$ & $\begin{array}{l}2.112 \\
(0.005,771.858) \\
0.172 \\
(0.000,64.994) \\
0.109 \\
(0.000,79.202) \\
\text { SADBE }\end{array}$ & $\begin{array}{l}40.009 \\
(1.242,4,553.721) \\
3.351 \\
(0.086,168.848) \\
2.178 \\
(0.007,581.668) \\
19.621 \\
(0.034,48,630.199) \\
\text { Intralesional corticosteroids }\end{array}$ & $\begin{array}{l}0.206 \\
(0.000,36.863) \\
0.017 \\
(0.000,2.726) \\
0.011 \\
(0.000,5.609) \\
0.095 \\
(0.000,241.194) \\
0.005 \\
(0.000,1.039) \\
\text { Calcineurin inhibitors }\end{array}$ & $\begin{array}{l}14.714 \\
(0.074,9,768.322) \\
1.212 \\
(0.002,801.753) \\
0.744 \\
(0.001,1,097.182) \\
7.078 \\
(0.056,4,335.967) \\
0.362 \\
(0.000,386.488) \\
75.129 \\
(0.062,686,938.467) \\
\text { DPCP }\end{array}$ & $\begin{array}{l}12.207 \\
(0.205,2,345.139) \\
1.024 \\
(0.008,119.510) \\
0.659 \\
(0.002,137.153) \\
5.937 \\
(0.025,4,074.079) \\
0.305 \\
(0.001,77.200) \\
60.989 \\
(0.121,255,505.701) \\
0.870 \\
(0.002,326.523) \\
\text { Minoxidil }\end{array}$ \\
\hline
\end{tabular}

Values are odds ratio (95\% confidence interval) (Aggregate Data Drug Information Software). Each cell represents the effect of the column-defining intervention relative to the row-defining intervention.

Table 3. Pooled odds ratios of the indirect, direct, and random effects standard deviation treatment comparisons of each head-to-head analysis included in the network

\begin{tabular}{|c|c|c|c|c|c|c|}
\hline Treatment 1 & Treatment 2 & $\begin{array}{l}\text { Indirect treatment } \\
\text { comparison }^{\mathrm{a}}\end{array}$ & $\begin{array}{l}\text { Direct treatment } \\
\text { comparison }^{\text {b }}\end{array}$ & Inconsistency factor $^{c}$ & $p$ value & $n^{\mathrm{d}}$ \\
\hline \multicolumn{7}{|l|}{ Mild disease } \\
\hline Calcineurin inhibitors & Intralesional corticosteroids & $-2.141(-10.933,6.148)$ & $-52.173(-162.360,-6.273)$ & $-50.169(-159.300,-2.474)$ & 0.028 & 1 \\
\hline Topical minoxidil & Prostaglandin analog & $-4.549(-15.894,5.983)$ & $1.278(-5.978,8.453)$ & $5.823(-6.769,19.088)$ & 0.297 & 1 \\
\hline Topical corticosteroids & Calcineurin inhibitors & $-0.933(-9.440,6.734)$ & $-56.625(-197.340,-5.297)$ & $-55.729(-195.580,-2.921)$ & 0.022 & 1 \\
\hline Topical corticosteroids & Intralesional corticosteroids & $1.047(-5.271,7.694)$ & $1.104(-3.599,5.771)$ & $0.062(-8.302,7.953)$ & 0.989 & 2 \\
\hline Topical corticosteroids & Topical minoxidil & $-2.554(-10.604,4.554)$ & $0.428(-6.350,7.223)$ & $3.003(-6.555,13.831)$ & 0.476 & 1 \\
\hline \multicolumn{7}{|l|}{ Moderate to severe disease } \\
\hline Topical corticosteroids & Topical tofacitinib & $2.371(-49.709,80.337)$ & $0.024(-7.251,7.342)$ & $-2.250(-80.456,50.124)$ & 0.958 & 1 \\
\hline
\end{tabular}

Values are odds ratio (95\% confidence interval) (Aggregate Data Drug Information Software, node-splitting analysis).

${ }^{a}$ Estimated using placebo or untreated control as a common comparator. ${ }^{b}$ Estimated directly from the trial using random-effects meta-analysis. ${ }^{c}$ Estimated using random-effects network meta-analysis. ${ }^{\mathrm{d}}$ Number of clinical trials making a direct comparison.

\section{Inconsistency Analysis}

Significant inconsistencies between the direct and indirect evidence were not detected with only one exception (Table 3). One study investigating the calcineurin inhibitor tacrolimus [12] compared with both topical and intra- lesional corticosteroids for treatment of mild disease resulted in a significant difference between direct and indirect evidence. Recent literature has suggested that the probability of inconsistency is higher in the case of fewer studies per treatment arm [13]. Thus, these results are not 
Table 4. Ranking of competing treatments for moderate to severe $\mathrm{AA}$ and mild AA

\begin{tabular}{lccc}
\hline Treatment & SUCRA & PrRank1 & $\begin{array}{l}\text { Mean } \\
\text { Rank }\end{array}$ \\
\hline Moderate to severe disease & & & \\
DPCP & 87.9 & 50.4 & 1.8 \\
Laser & 77.9 & 26.9 & 2.6 \\
Topical minoxidil & 55.5 & 4.8 & 4.1 \\
Topical corticosteroids & 50.1 & 11.0 & 3.4 \\
SADBE & 49.7 & 1.9 & 4.5 \\
Topical tofacitinib & 47.6 & 5.0 & 4.7 \\
Control & 16.2 & 0.00 & 6.9 \\
\hline Mild disease & & & \\
Intralesional corticosteroids & 78.9 & 36.2 & 2.5 \\
Topical corticosteroids & 67.9 & 24.6 & 3.8 \\
Prostaglandin analogs & 67.1 & 21.9 & 3.3 \\
DPCP & 63.4 & 22.0 & 3.6 \\
Topical minoxidil & 61.2 & 11.1 & 3.7 \\
SADBE & 35.0 & 3.9 & 5.5 \\
Calcineurin inhibitors & 12.3 & 0.6 & 7.1 \\
Control & 21.7 & 0.00 & 6.5 \\
\hline
\end{tabular}

SUCRA, surface under the cumulative ranking curve; PrRank1, probability of a treatment to ranking first.

surprising; however, the possibility of an outlier cannot be eliminated without investigating additional studies [13].

\section{Ranking of Treatments}

In the case of moderate to severe disease, DPCP was ranked the most likely to produce responders (87.9\%), followed by laser (77.9\%), topical minoxidil (55.5\%), topical corticosteroids (50.1\%), and SADBE (49.7\%) according to SUCRA (surface under the cumulative ranking curve) (Table 4 ). With a $47.6 \%$ probability, topical tofacitinib was ranked the least likely active treatment to produce responders. For mild disease, intralesional corticosteroids were ranked the most likely to produce responders $(78.9 \%)$, followed by topical corticosteroids (67.9\%), prostaglandin analogs (67.1\%), DPCP $(63.4 \%)$, topical minoxidil (61.2\%), and SADBE (35.0\%) according to SUCRA (Table 4).

\section{Discussion}

Based on network meta-analysis and meta-analysis results, DPCP, laser, topical minoxidil, topical corticosteroids, and SADBE treatment were significantly more ef- fective (OR) in the case of moderate to severe scalp AA compared with untreated or placebo-treated controls. Likewise, in the case of mild disease, intralesional corticosteroid treatments (network meta-analysis) and topical corticosteroids (meta-analysis) demonstrated significantly greater OR of attaining responders compared with control treatments. Based on SUCRA, intralesional corticosteroids were ranked with the highest probability of successful treatment (based on number of responders) followed by topical corticosteroids, prostaglandin analogs, DPCP, topical minoxidil, SADBE, and calcineurin inhibitors for the treatment of mild disease. For moderate to severe disease, SUCRA ranked DPCP with the highest probability of success followed by laser, topical corticosteroids, topical minoxidil, SADBE, and topical tofacitinib. Use of network meta-analysis not only allowed for ranking of treatments but was also a method of analyzing direct and indirect evidence. Use of both techniques is considered beneficial as direct evidence is considered higher quality and not subject to issues of similarity/transitivity but may result in similar bias not present in indirect evidence [14].

Nonetheless, confidence in SUCRA rankings should be interpreted according to quality of evidence [15]. Quality of evidence was found to vary from moderate to very low. Evidence was downgraded to very low in cases where data was extracted from nonrandomized trials or a mixture of randomized and nonrandomized trials. Therefore, although observational studies have been important in identifying potential treatments, this analysis highlights the need for randomized controlled trials to confirm those results. Randomized, controlled trials could be especially important in identifying a role for potential new treatments such as oral and topical tofacitinib, ruxolitinib, and platelet-rich plasma. A limitation of the current analysis is the inability to include oral tofacitinib [16] due to a lack of controlled studies. With only off-label treatments currently available, investment in randomized controlled trials could make a significant difference for AA patients. A second point to consider is the inclusion of both half-head and non-half-head studies. As many AA treatments are local, use of half-head studies has been considered beneficial for the detection of spontaneous remissions. Despite this, there is also evidence of a systemic effect of localized application leading to the possibility of an underestimated treatment effect [17].

Similar to recent treatment recommendations [18], this analysis also suggests that severity of disease should influence front-line treatment options. For patients with mild and patchy hair loss, either intralesional or topical 
corticosteroids might be a first-choice treatment which aligns with current prescribing practices. In comparison, patients with $>50 \%$ scalp hair loss might benefit from progressing straight to DPCP which is also consistent with the literature that suggests efficacy for severe AA [19] but not for use in cases of mild AA [20]. Additionally, treatment choice should also consider the individual patient. For instance, not all patients are candidates for DPCP use, including pregnant women due to possible mutagenic properties of a possible contaminant [21] as well as younger patients with the possible adverse effects such as blistering, severe irritation, hyperpigmentation, cervical and occipital lymphadenopathy, urticaria, facial swelling, erythema multiforme and severe pruritus $[22,23]$, and development of multiple lentigines [24]. Further, although efforts were made to divide studies by the severity of disease, there were still differences within the two analyses. For example, one investigation of tofacitinib included only patients diagnosed with AU [25], which has been documented with increased recalcitrance to treatment [26]. Thus, the lower ranking of topical tofacitinib in this analysis might be a result of the treatment population versus the effectiveness of the drug.

Use of responders with $>75 \%$ regrowth and cosmetic acceptability was chosen as a measure of efficacy due to the nature of the disease. Partial regrowth was not considered as patients who still require use of hair pieces, camouflage, hats, and other head coverings are not considered treatment successes [27].

Overall, despite being off-label treatments, intralesional corticosteroids and topical corticosteroids were found to lead to a significantly greater number of responders for mild disease and DPCP, SADBE, topical minoxidil, topical corticosteroids, and laser were found to lead to a significantly greater number of responders for moderate to severe disease. Due to the limited number of trials, the current analysis included both randomized and nonrandomized trials. Combination treatment and relapse rates were not analyzed. Despite these limitations, evidence is provided for the use of off-label treatments for mild and moderate to severe AA.

\section{Statement of Ethics}

The literature search was defined a priori in an internal protocol and registered on PROSPERO under the code CRD42018105258 (online suppl. Table S1). IRB approval was not necessary as this was a review study.

\section{Disclosure Statement}

A.K.G., J.L.C., K.A.F., N.H.S., and B.M.P. have no conflicts of interest to declare. V.P. undertakes advisory work for Pfizer, AbbVie, Janssen, UCB, Novartis, Almirall, and Celgene. He has received departmental support from AbbVie, Bausch Health, Celgene, Janssen, LEO Pharma, Lilly, NAOS, Novartis, Pfizer, PierreFabre, and Sanofi. A.T. is a consultant for Pfizer and a primary investigator for Incyte, Aclaris, Eli Lilly, and Nutrifol.

\section{Author Contributions}

A.K. Gupta, MD, PhD: substantial contributions to the conception and design of the work, analysis and interpretation of data, and revising the work critically for important intellectual content. J.L. Carviel, PhD: substantial contributions to the design of the work, acquisition, analysis, and interpretation of data, and drafting the work critically for important intellectual content. K.A. Foley, $\mathrm{PhD}$ : substantial contributions to the acquisition, analysis, and interpretation of data, and revising the work critically for important intellectual content. N.H. Shear, MD, B.M. Piraccini, MD, V. Piguet, $\mathrm{MD}, \mathrm{PhD}$, and $\mathrm{A}$. Tosti, MD: substantial contributions to the analysis and interpretation of data, and revising the work critically for important intellectual content.

All authors gave final approval of the version to be published and agreed to be accountable for all aspects of the work in ensuring that questions related to the accuracy or integrity of any part of the work are appropriately investigated and resolved.

\section{References}

1 Gilhar A, Kalish RS. Alopecia areata: a tissue specific autoimmune disease of the hair follicle. Autoimmun Rev. 2006 Jan;5(1):64-9.

2 McElwee K, Freyschmidt-Paul P, Ziegler A, Happle R, Hoffmann R. Genetic susceptibility and severity of alopecia areata in human and animal models. Eur J Dermatol. 2001 Jan-Feb; 11(1):11-6.

3 Gupta AK, Carviel JL, Abramovits W. Efficacy of tofacitinib in treatment of alopecia universalis in two patients. J Eur Acad Dermatol Venereol. 2016 Aug;30(8):13738.

4 Peters EM, Liotiri S, Bodó E, Hagen E, Bíró T, Arck PC, et al. Probing the effects of stress mediators on the human hair follicle: substance P holds central position. Am J Pathol. 2007 Dec;171(6): 1872-86.

5 McElwee KJ, Gilhar A, Tobin DJ, Ramot Y, Sundberg JP, Nakamura M, et al. What causes alopecia areata? Exp Dermatol. 2013 Sep; 22(9):609-26.

6 van der Steen P, Traupe H, Happle R, Boezeman J, Sträter R, Hamm H. The genetic risk for alopecia areata in first degree relatives of severely affected patients. An estimate. Acta Derm Venereol. 1992 Sep;72(5):373-5.

7 McDonagh AJ, Tazi-Ahnini R. Epidemiology and genetics of alopecia areata. Clin Exp Dermatol. 2002 Jul;27(5):405-9. 
8 Biran R, Zlotogorski A, Ramot Y. The genetics of alopecia areata: new approaches, new findings, new treatments. J Dermatol Sci. 2015 Apr;78(1):11-20.

9 The Cochrane Collaboration. Assessing risk of bias in included studies [Internet]. Available from: http://methods.cochrane.org/bias/ assessing-risk-bias-included-studies.

10 van Valkenhoef G, Tervonen T, Zwinkels T, de Brock B, Hillege H. ADDIS: A decision support system for evidence-based medicine. Decis Support Syst. 2013 May;55(2): 459-75.

11 Coleman CI, Phung OJ, Cappelleri JC, Baker WL, Kluger J, White CM, et al. Use of mixed treatment comparisons in systematic reviews [Internet]. Agency for Healthcare Research and Quality (USA); 2012 [cited 2018 Nov 19]. Available from: https://www.ncbi.nlm.nih. gov/books/NBK107325/.

12 Kuldeep C, Singhal H, Khare AK, Mittal A, Gupta LK, Garg A. Randomized comparison of topical betamethasone valerate foam, intralesional triamcinolone acetonide and tacrolimus ointment in management of localized alopecia areata. Int J Trichology. 2011 Jan; $3(1): 20-4$.

13 Veroniki AA, Vasiliadis HS, Higgins JP, Salanti G. Evaluation of inconsistency in networks of interventions. Int J Epidemiol. 2013 Feb;42(1):332-45.
14 Bafeta A, Trinquart L, Seror R, Ravaud P. Reporting of results from network meta-analyses: methodological systematic review. BMJ. 2014 Mar;348 mar11 5:g1741.

15 Mbuagbaw L, Rochwerg B, Jaeschke R, HeelsAndsell D, Alhazzani W, Thabane L, et al. Approaches to interpreting and choosing the best treatments in network meta-analyses. Syst Rev. 2017 Apr;6(1):79.

16 Almutairi N, Nour TM, Hussain NH. Janus kinase inhibitors for the treatment of severe alopecia areata: an open-label comparative study. Dermatology. 2019;235(2):130-136.

17 Khandpur S, Sharma VK, Sumanth K. Topical immunomodulators in dermatology. J Postgrad Med. 2004 Apr-Jun;50(2):131-9.

18 Strazzulla LC, Wang EH, Avila L, Lo Sicco K, Brinster N, Christiano AM, et al. Alopecia areata: an appraisal of new treatment approaches and overview of current therapies. J Am Acad Dermatol. 2018 Jan;78(1): $15-24$.

19 Ro BI. Alopecia areata in Korea (1982-1994). J Dermatol. 1995 Nov;22(11):858-64.

20 Tosti A, De Padova MP, Minghetti G, Veronesi $\mathrm{S}$. Therapies versus placebo in the treatment of patchy alopecia areata. J Am Acad Dermatol. 1986 Aug;15(2 Pt 1):209-10.

21 Wilkerson MG, Connor TH, Henkin J, Wilkin JK, Matney TS. Assessment of diphenylcyclopropenone for photochemically induced $\mathrm{mu}$ - tagenicity in the Ames assay. J Am Acad Dermatol. 1987 Oct;17(4):606-11.

22 Pan R, Liu J, Xuan X, Li B. Chinese experience in the treatment of alopecia areata with diphenylcyclopropenone. J Dermatol. 2015 Feb; 42(2):220-1.

23 Chiang K, Atanaskova Mesinkovska N, Amoretti A, Piliang MP, Kyei A, Bergfeld WF. Clinical efficacy of diphenylcyclopropenone in alopecia areata: retrospective data analysis of 50 patients. J Am Acad Dermatol. 2014 Sep; 71(3):595-7.

24 Tosti A, Piraccini BM, Misciali C, Vincenzi C. Lentiginous eruption due to topical immunotherapy. Arch Dermatol. 2003 Apr;139(4): 544-5.

25 Bokhari L, Sinclair R. Treatment of alopecia universalis with topical Janus kinase inhibitors - a double blind, placebo, and active controlled pilot study. Int J Dermatol. 2018 Dec; 57(12):1464-70.

26 Jang YH, Hong NS, Moon SY, Eun DH, Lee WK, Chi SG, et al. Long-Term Prognosis of Alopecia Totalis and Alopecia Universalis: A Longitudinal Study with More than 10 Years of Follow-Up: Better than Reported. Dermatology. 2017;233(2-3):250-6.

27 Delamere FM, Sladden MM, Dobbins HM, Leonardi-Bee J. Interventions for alopecia areata. Cochrane Database Syst Rev. 2008 Apr;(2): CD004413. 